Reprinted with permission from: Weed Technology, 1991. 5:221-228.

Published and copyrighted by: Weed Science Society of America. http://www.wssa.net

\title{
Format of a weed control database ${ }^{1}$
}

\author{
G. W. MUELLER-WARRANT \\ The author is with the Res. Agron., Agric. Res. Serv., USDA, Nat. Forage Seed Prod. Res. Cent., 3450 SW Campus \\ Way, Corvallis, OR 97331-7102.
}

\begin{abstract}
:
Access to detailed descriptions of the effects of applying specific rates of herbicides to crops and weeds in various growth stages is hampered by the format in which the relevant information is stored. Compared to traditional formats of journal articles and herbicide registration labels, computer database systems could easily cross-reference data from large numbers of experiments and answer specific questions concerning herbicide performance under particular conditions. Availability of this type of information could have far-reaching consequences for herbicide users, consultants, researchers, and regulators. A preliminary format for storing weed control information in IBM-PC compatible computers was developed, including procedures to enter data and retrieve information. Weed control efficacy or crop injury data for all rates of a herbicide or tank-mixture applied at a specific growth stage in a single test are used to generate dose/response equations by means of regression analysis routines. The best fitting of these equations is then used to estimate herbicide rates that would provide ten categories of control, ranging from a "no observable effect level" (NOEL) up to complete control. Rates are estimated only for those categories either within or bordering the range of the observed data, the remaining categories are empty. The estimated rates are stored in the database, along with the original data and other qualifying information. Access to information is organized around searches for a single herbicide, plant species, or pair of species. Search output is presented in a tabular format listing species, growth stage, herbicide name, and herbicide rates for the ten categories: NOEL, 10, 30, 50, 70, 83, 90, 95, 98, and 100\% control or injury.
\end{abstract}

\footnotetext{
${ }^{1}$ Received for publication May 21, 1990, and in revised form Dec. 6, 1990. Contribution of Agric. Res. Serv., USDA, in cooperation with the Agric. Exp. Stn., Oregon State Univ. (Tech. Paper No. 8655 of the latter).
} 


\section{Additional words:}

Personal computer, regression analysis, dose/response equations, growth stage, NOEL.

\section{Introduction}

\section{Current practices}

Efficient access to information is vital to active research programs and is an important link between researchers, users, consultants, and regulators. Traditional methods used to summarize weed control information include such formats as herbicide registration labels, articles in peer-reviewed journals, extension handouts, and research project reports. Although these formats are useful, they often are poorly indexed and cross-referenced, and searching through them for specific data can be a tedious process. A personal computer that stores weed control information in an accessible database could improve the efficiency with which weed control questions are answered. Another benefit could be that the database would highlight gaps in the existing data. While these benefits would be most pronounced if the database were regional or national in scope, it could be quite useful even if localized in scope to a few collaborating researchers at a single institution.

\section{Obstacles to developing computer-based systems}

Creation of a computer database system well suited to answering weed control questions is no trivial undertaking. Details of its structure will impose fundamental limits on data entry, access speed, information quality, and general ease of use. The central issue in creating a weed control database is how to efficiently represent the susceptibilities of different species and growth stages of plants to a variety of herbicides under various environmental conditions. Resolution of this issue can be aided by examination of the traditional mechanisms of presenting weed control information.

Publications such as the Pacific Northwest Weed Control Handbook include tables of susceptibility ratings of a number of weeds to various herbicides (2). Specific herbicide rates are usually not indicated, nor is the growth stage of the weed. Instead, the reader is referred to supplemental text and/or the herbicide label for such details. Susceptibility may simply be rated in values of yes or no; alternatively, herbicide effectiveness may be listed as excellent, good, fair, or poor. Expansion of the rating scale in such a table is one way to increase the value of the data, but highly specific control ratings must be tied to exact herbicide rates, weed growth stages, and environmental conditions. The approach taken by the developers of WEEDS, a computer-based system for recommending herbicides, was to transfer traditional susceptibility ratings into a computer file format (4). In their program, the relationship between a weed species and a registered herbicide treatment consists of a single control rating with eight possible values. Neither herbicide rates nor weed growth stages are explicitly present in their program, although such information 
is available in herbicide labels and is an implicit part of whether a treatment has been registered.

The opposite extreme in weed control information is maintenance of the full set of raw data. This type of control information is tied to the rates of herbicides actually tested on a given species at a particular growth stage. A compendium of raw data from all relevant experiments is one possible format for a computer database system. Indeed, the Electronic Data Exchange system represents a step toward that format (7). A serious limitation to such a format is that raw data from unrelated tests inherently span wide and nonstandardized ranges in herbicide rates and plant responses. Some processing of raw data must occur before weed control information can be presented in a succinct format that facilitates comparisons between species, herbicides, and environmental conditions. If only raw data are stored in the database, then such processing must occur each time the information is accessed.

There are two advantages to performing this processing during data entry: first, the speed of information retrieval from the database is greatly increased; and second, the professional judgment of the researcher can be used to decide the method to represent the dose/response relationship, instead of relying solely on programmed algorithms. There are also two major disadvantages to this format: first, data entry is slowed down by the need to choose the appropriate representation; and second, the regression equation selected may introduce an unwanted degree of distortion between the raw data and the processed information. The primary obstacle to developing a useful weed control database system is finding a method, which is relatively, even if not entirely, free of distortion for transforming raw data into the processed form actually stored and retrieved.

The objective of this program was to create a database structure that would begin to bridge the gap between raw data collected by researchers on one extreme and lists of species controlled by herbicides as given in weed control handbooks and herbicide labels on tbe other. It does not include registration status information and was not intended to provide all answers to any possible questions. Rather, its purpose was to define a reasonable framework around which further progress could be made toward a distant goal of providing herbicide users and regulators with much more detailed information on the performance of herbicide treatments under their consideration than what is currently available to them. This version of the database was designed to be primarily local in scope, with the possibility of regional use when restricted to the interests of specific projects. It lacks several components that would be necessary in a truly national format.

\section{Database format}

The database is organized as seven tables stored in three separate files. Two of the tables grow as data are added to the database (CNTLRATE and ENTRYLOG), whereas the other tables are small, temporary, and used only in the data entry process. The database includes 27 fields within each record in the table CNTLRATE to store treatment details and a species dose/response behavior for a primary herbicide. The primary herbicide is either the only herbicide used in a treatment or it is one selected component of a tankmixture. Dose/response equations are developed for treatments with various rates of a

Page 3 of 12 
single herbicide or a tank-mixture in which the proportions of two herbicides remain constant. Designation of primary and secondary components of a tank-mixture is at the user's discretion, but only the primary herbicide is directly used in current database search routines. The database also stores descriptions of as many as three background herbicides applied at constant rates. Possible synergism or antagonism between the primary herbicide and these background treatments is not explicitly tested by the program, but would be apparent when the information is retrieved. The presence or absence of a secondary herbicide in a tank-mixture and any additional background constant treatments is reported in abbreviated format during database access. If ability to search for either herbicide component of a tank-mixture is deemed important, the same data can simply be entered twice, changing which is designated as primary and which as secondary.

The 27 fields in the table CNTLRATE hold the information summarizing the response of a single species to all rates of an herbicide applied in a specific test. Bayer code format (e.g., $\#^{2}$ LOLMU) is used to identify plant species. Herbicides are identified by common names of the primary and secondary herbicides, herbicide formulation, and the proportion of a tank-mixture that is the primary herbicide. Growth stage of the species when treated is specified in an arbitrary format defined within the program. Treatments are further characterized by date of application, geographic location of test (county, state), evaluation timing in days after application, sequential reference number, and units of measurement. Standardized herbicide rates for the ten categories of weed control or crop injury are estimated from the selected regression equation. Also included are the original raw data and information on any other herbicides that were applied at constant rates before, during, or after application of the primary (and secondary, if also present) herbicide. Growth stage system includes pre-emergence treatments, which are described using the negative value of the number of days between application and weed emergence in untreated checks.

The weed control categories are $2,10,30,50,70,83,90,95,98$, and $100 \%$ control or injury. The ten standardized categories or levels of control were selected to represent a wide range of plant responses, corresponding to conditions of essentially no injury ( $2 \%$ or NOEL); minor but detectable damage (10\%); major damage when viewed as a crop (30\%); mid-point of the response curve (50\%); fair weed control (70\%); fair to good control (83\%); good control (90\%); good to excellent control (95\%); excellent control (98\%); and total control $(100 \%)$. The NOEL was arbitrarily assigned to a rating of $2 \%$ injury on the basis of such damage being indistinguishable from zero under most practical test conditions.

Another possible structure to hold the dose/response information would be a scale with 101 categories between 0 and $100 \%$ control. In such a format, it would even be possible to dispense with the regression routines and simply enter the real herbicide rates for categories equal to the observed control. Problems with this format include the presence of many empty categories between the observed levels of control, the natural scatter of

\footnotetext{
${ }^{2}$ Letters following this \# symbol are a WSSA-approved computer code from Composite List of Weeds, Revised 1989. Available from WSSA, 309 W. Clark St., Champaign, IL 61820.
} 
raw data (random error), and the difficulty in presenting such a large number of treatment categories on the computer screen or even in printed tables. The ten categories selected for this database provide a reasonably complete description of plant response within the boundaries of what can be displayed on a single line of a computer screen.

The table ENTRYLOG is used to store information pertinent to an entire experiment. One record in ENTRYLOG can therefore be linked to as many records in CNTLRATE as there are treatments in an experiment. The nine fields in the table ENTRYLOG consist of the name of the user entering the data, the name of the researcher who performed the experiment, a description of where to find a published version of the data, the publication date, the geographic location of the experiment as city, county, and state, a unique sequential reference number, and the date on which the data were entered into the database.

\section{Data entry procedures}

\section{Specifying sites, weeds, and herbicides}

The process of entering data begins with the user describing the source of the data. This description is used to fill in the fields of the table ENTRYLOG. The primary purpose of this action is to provide subsequent users of the database with a way to find a published version of the results from the original experiment. This information is linked to all data entered from that test through use of a unique sequential reference number generated by the program. Data is then entered one species at a time for all rates of a single herbicide or of a tank-mixture. The user enters species name, herbicide name or names, how many herbicide rates were tested, environmental conditions, and growth stage of the species in question before entering the specific herbicide rates and plant injury data. Grass growth stage is recorded using a modified combination of Zadoks' scale for cereals (10) and Robson's scale for perennial ryegrass (9). Broadleaf growth stage is recorded using a modified version of that given by Lutinan and Tucker (6). The growth stages are described in on-line help screens included with the program. The presence of environmental stresses that may have influenced the results is also recorded using an arbitrary scale described on screen. The help screens are provided through use of terminate and stay resident (TSR) programs CAPTURE.COM and HELP.COM $(3,8)$. Information screens that can be popped up from within the program include descriptions of the growth staging systems, the stress factor rating system, and names of species and herbicides already entered in the database.

\section{Entering and transforming data}

Herbicide rates and percent crop injury or weed control are entered and edited in a small onscreen table. Where only one herbicide rate is available, the program automatically generates a second data point of near-zero weed control for a near-zero herbicide rate, thus providing the minimum number of data points (two) necessary to calculate regressions. Near zero is defined as $0.5 \%$ injury or control at a herbicide rate linearly reduced from the observed data to same degree. Near-zero values are used rather than true

Page 5 of 12 
zero because several of the regression routines fail at the origin. If values of zero weed control for a zero herbicide rate are entered by the user, they also are converted into nearzero values. Herbicides that have either total efficacy (100\% control) or complete tolerance ( $0 \%$ injury) at all rates tested are stored in the database without further processing as either the lowest rate that effects $100 \%$ control or the highest rate that results in no detectable injury.

The actual crop injury or weed control data are subjected to linear, logistic, asymptotic, and power function regressions versus herbicide rate as given by Little and Hills (5) and described in Table 1. Other more general dose/response equations exist, such as the modified sigmoidal equation proposed by Brain and Cousens (1), but would be more difficult to implement in this programming language and not improve the goodness of the fit with fewer than four data points.

Table 1. Dose/response regression equations used to calculate herbicide rates for control categories.

\begin{tabular}{|c|c|c|}
\hline Type & Formula $^{\mathrm{a}}$ & Regression method \\
\hline Linear & $\mathrm{Y}=\mathrm{A}+\mathrm{B} * \mathrm{X}$ & Linear regression of observed control against herbicide rate. \\
\hline Logistic & $\begin{array}{l}Y=100 \% * \\
\operatorname{Exp}(A+B * X) / \\
(1+\operatorname{Exp}(A+B * X))\end{array}$ & $\begin{array}{l}\text { Linear regression of transformed control against herbicide rate } \\
\text { using the transformation } \mathrm{Y}^{\prime}=\log (.01 * \mathrm{Y} /(1-.01 * \mathrm{Y})) \text { to obtain } \\
\text { values of } \mathrm{A} \text { and } \mathrm{B} \text {. }\end{array}$ \\
\hline Asymptotic & $\mathrm{Y}=\mathrm{C}-\mathrm{A} * \mathrm{~B}^{\mathrm{X}}$ & $\begin{array}{l}\text { Linear regression of transformed control against herbicide rate } \\
\text { using the transformation } \mathrm{Y}^{\prime}=\log (\mathrm{C}-\mathrm{Y}) \text {, where } \mathrm{C} \text { is the value } \\
\text { of the asymptote, to obtain values of } \log (\mathrm{A}) \text { and } \log (\mathrm{B}) \text {. } \\
\text { Program does not optimize the asymptote, but user can repeat- } \\
\text { edly change it and recalculate. }\end{array}$ \\
\hline $\begin{array}{l}\text { Power } \\
\text { function }\end{array}$ & $\mathrm{Y}=\mathrm{A} * \mathrm{X}^{\mathrm{B}}$ & $\begin{array}{l}\text { Linear regression of transformed control against transformed } \\
\text { herbicide rate, using transformations } \mathrm{Y}^{\prime}=\log (\mathrm{Y}), \mathrm{X}^{\prime}=\log \\
\text { (X) to obtain values for } \log (\mathrm{A}) \text { and } \mathrm{B} \text {. }\end{array}$ \\
\hline
\end{tabular}

${ }^{\mathrm{a}}$ Control $\mathrm{Y}$ expressed in terms of percentage and herbicide rate $\mathrm{X}$ expressed in arbitrary units such as $\mathrm{kg} \mathrm{ha}^{-1}$, gm $\mathrm{ha}^{-1}$, lbs acre ${ }^{-1}$, oz acre ${ }^{-1}$.

Based on these four regressions, rates of herbicide required to produce the ten standardized categories of control are calculated, and subsets of these ten rates are displayed on screen (Figure 1). The user is then asked to select which of these four alternative equations should be used to transform the data for storage in the database. All ten values from the NOEL rate up to the complete $(100 \%)$ control rate would be calculated only if the observed data spanned the full range ( 2 to $100 \%$ ). Since observed data typically do not cover such a wide range in plant responses, the program restricts the calculation range to the nearest standardized control categories immediately above and below the highest and lowest control values actually observed. The remaining categories are left empty to indi- 
cate that those levels of control fell substantially outside the observed range. When determining the appropriate range of control categories over which to estimate herbicide rates to store in the database, the program excludes the arbitrary near-zero value introduced if only one herbicide rate was tested. This means that data for a herbicide applied at only one rate will generally be transformed into two estimated rates in the database. However, if the observed control from a single-rate treatment exactly equaled any one of the ten categories of control, then estimated herbicide rates would be stored for that category and the neighboring ones immediately above and below it. If the user is dissatisfied with all four equations, options exist to change the calculation boundaries (increasing or decreasing the range), alter the value of the asymptote, re-enter the raw data, or abort the data entry process. Otherwise, the data are stored in the database based on the selected transformation.

Figure 1. Method used to display category rates calculated from alternative dose/response regression equations. Empty categories are indicated by use of the symbol -0- the default null value in RBASE.

\begin{tabular}{|c|c|c|c|c|c|c|c|}
\hline Weed & Model: & Linear & Logistic & Asymptote & Power & FENOXAPROP & LOLMU \\
\hline control & $\mathrm{R}^{\wedge} 2:$ & 0.873 & 0.6903 & 0.8637 & 0.9837 & \multicolumn{2}{|c|}{ Real Data } \\
\hline level & ‘Adj'R^2: & 0.873 & 0.5382 & 0.9205 & 0.8087 & \multirow{4}{*}{$\begin{array}{c}\text { Herbicide } \\
\text { rate } \\
(\mathrm{Lb} / \mathrm{A})\end{array}$} & \multirow{4}{*}{$\begin{array}{c}\text { Weed } \\
\text { control } \\
(\%)\end{array}$} \\
\hline avg. & Active & \multirow{2}{*}{\multicolumn{4}{|c|}{ Predicted herbicide rates $(\mathrm{Lb} / \mathrm{A})$}} & & \\
\hline$(\%)$ & range & & & & & & \\
\hline 2 & & $-0-$ & $-0-$ & $-0-$ & $-0-$ & & \\
\hline 10 & $* 2$ & $-0-$ & 0.0548 & 0.0123 & 0.0212 & 0.0008 & 0.5 \\
\hline 30 & $* 3$ & 0.0695 & 0.1193 & 0.0592 & 0.0728 & 0.05 & 30. \\
\hline 50 & $* 4$ & 0.1433 & 0.1598 & 0.1219 & 0.1294 & 0.1 & 50. \\
\hline 70 & $* 5$ & 0.2171 & 0.2003 & 0.2171 & 0.1889 & 0.15 & 57. \\
\hline 83 & $* 6$ & 0.265 & 0.2356 & 0.323 & 0.2287 & 0.2 & 53. \\
\hline 90 & & $-0-$ & $-0-$ & $-0-$ & $-0-$ & 0.25 & 80. \\
\hline 95 & & $-0-$ & $-0-$ & $-0-$ & $-0-$ & & \\
\hline 98 & & $-0-$ & $-0-$ & $-0-$ & $-0-$ & \multirow{2}{*}{\multicolumn{2}{|c|}{ Asymptote $=100$. }} \\
\hline 100 & & $-0-$ & $-0-$ & $-0-$ & $-0-$ & & \\
\hline \multicolumn{8}{|c|}{ Select transformation to use for saving data to WEEDATA } \\
\hline & & (1) & \multicolumn{3}{|c|}{ Linear regression model } & & \\
\hline & & (2) & \multicolumn{3}{|c|}{ Logistic transformation } & & \\
\hline & & (3) & \multicolumn{3}{|c|}{ Asymptotic curve model } & & \\
\hline & & (4) & \multicolumn{3}{|c|}{ Power function model } & & \\
\hline & & $(5)$ & \multicolumn{3}{|c|}{ Restrict range of control \% } & & \\
\hline & & (6) & \multicolumn{3}{|c|}{ Change value of asymptote } & & \\
\hline & & (7) & \multicolumn{3}{|c|}{ None OK yet; Re-enter data } & & \\
\hline & & $(8)$ & \multicolumn{3}{|c|}{ Exit w/o saving this data } & & \\
\hline
\end{tabular}



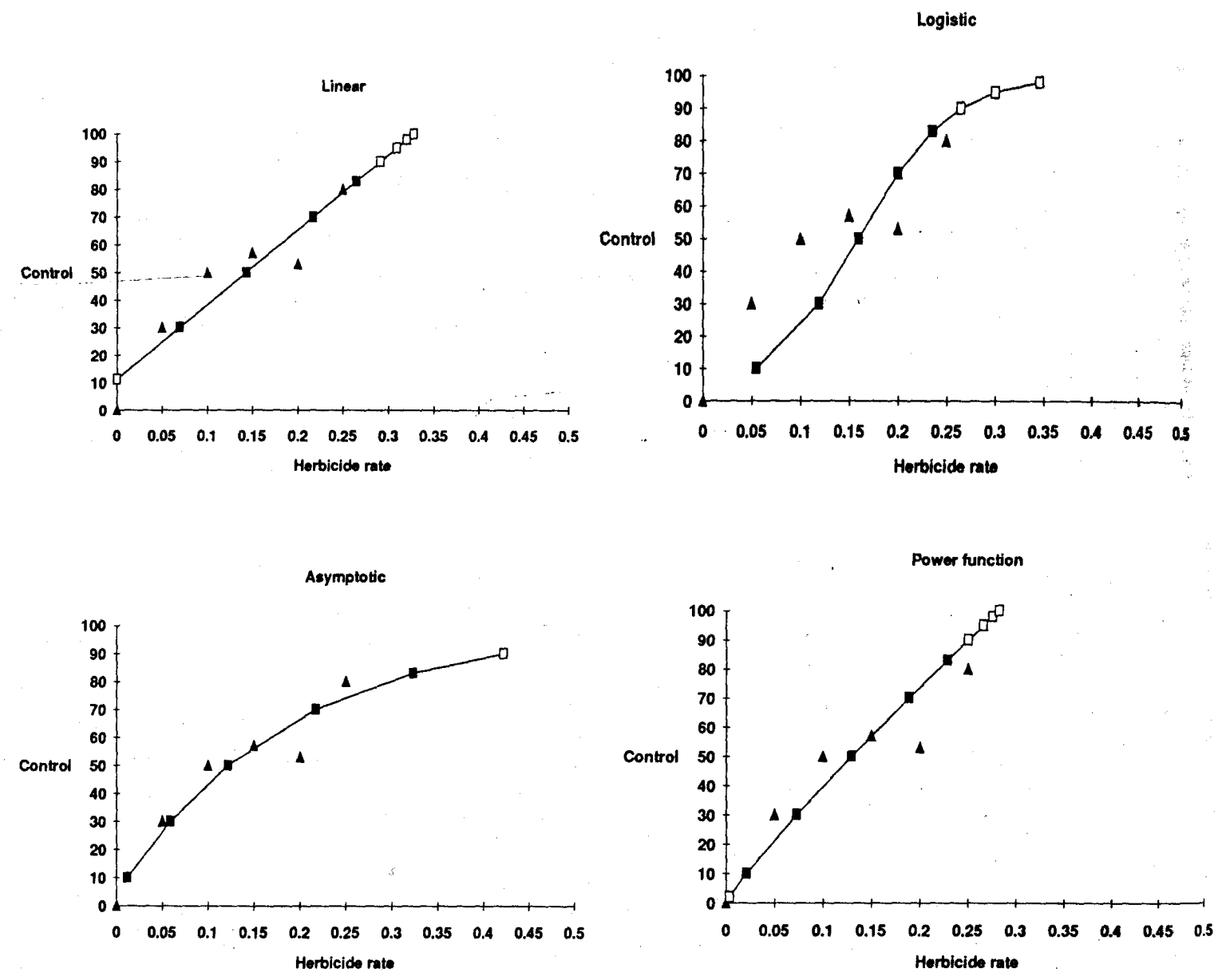

Figure 2, Graph of sample data and category rates calculated from linear, logistic, asymptotic, and power function regression equations. Closed triangles are real data points, closed rectangles are calculated points (herbicide rates for specific control categories) within the boundary region of this data set, and open rectangles are potentially calculated points outside the boundary region.

Formulas for the four regression equations are given in Table 1, and examples are graphed in Figure 2 using the same data presented in Figure 1. Linear regression is the simplest model, and may do an adequate job of extrapolating to neighboring standardized control categories for treatments with only one or two herbicide rates, particularly if the weed control or crop injury ratings do not fall into the extreme categories. Possible benefits from using the logistic equation instead of the alternative functions relate to its inherent S-shape. There is a lag phase in response to low rates of herbicides, similar to what may be seen in the field; response near the midpoint of the curve is approximately linear; and the curve asymptotically approaches full control at higher herbicide rates. Because of singularities in the inverse of the logistic equation, neither the lower nor upper limits $(0$ and $100 \%$ ) can be reached. The asymptotic model often fits data well in those cases where one or more of the rates tested provided nearly complete control and the asymptote 
are assigned a value near or slightly above $100 \%$. This equation fails if values of any of the observed data or calculated control categories equal or exceed the asymptote. The power function is included to provide an alternative equation in those cases where none of the others fit the data particularly well. All four of these equations make better biological sense than quadratic, cubic, or quartic functions, which possess inflection points usually without any biological explanation. A possible modification of the logistic equation in which its asymptotes are allowed to take on arbitrary values other than 0 and $100 \%$ would be capable of fitting most data extremely well, but would require more data points to solve for the coefficients and would be harder to program. This type of equation would be similar, although not identical to the sigmoidal equations discussed by Brain and Cousens (1).

Coefficients of the logistic, asymptotic, and power function equations are calculated from linear regressions of logarithmic transformations of the data. The program calculates two coefficients of determination (R-squared values) for each of the four curves, one for the transformed data used in each regression and the other for the difference between observed and predicted values following inverse transformation back to the original, linear units of measurement. This second value is shown as the "adjusted" R-squared value, and provides a common basis to compare the four analyses. Cases involving only a single data point will frequently cause some of the calculations to fail, and may generate Rsquared values above 1 or below 0 . Such equations should not be selected as the standardization method unless the estimated herbicide rates are reasonable and only the Rsquared calculations have failed.

\section{Database query}

\section{Developed routines}

Retrieval of stored information is based on searching the database within certain restrictions entered by the user. The current version of the database access routines allows searches for six different types of information. Output of the searches can be directed to the computer screen, the printer, both, or a DOS file in ASCII text format. Each record matching search criteria is retrieved individually and presented sequentially on screen, generally at one line per record. No attempt is made to summarize the separate records beyond the logic involved in their order of retrieval.

The first type of search is for all herbicide treatments that have provided fair or better control of a single species. Output is sorted by primary herbicide name, plant growth stage at treatment, and order of entry into the database. The second search format is more restricted; output being limited to tests in which the selected herbicide was applied to two species of interest (Figure 3). One species would typically be a crop and the other a weed of interest in that crop. This would simultaneously provide both crop tolerance and weed control efficacy information from all tests in which the herbicide was applied to both species. The third type of search is more general, providing all information available on two species. Output is sorted by ENTRYLOG reference number, growth stage, and herbicide, and includes all herbicides that were evaluated on either species, no matter how poorly

Page 9 of 12 
they performed. The fourth type of search is restricted to a single herbicide, and provides a list of all species against which this herbicide provided fair or better control (70 to $100 \%$ ), as well as providing the rate response information for each of these species in this control range. The fifth type of search calls up all of the ENTRYLOG information for a single reference number, and could be used to check on the details of who conducted an experiment, where it was performed, and where it was published. The sixth type of search is based on the ENTRYLOG reference number and provides a complete listing of all species' response data to all herbicides tested in a single trial, assuming each entry corresponds to one complete experiment.

Figure 3. Display screen for search option 2 (two species, one herbicide).

\begin{tabular}{|c|c|c|c|c|c|c|c|c|c|c|c|c|c|c|}
\hline SPP & GROWTH & I Mix/Seq/ & REF & RATE & NOEI & MIN & MAJ & GR50 & FAIR & F-G & GOOD & G-E & EXCL & TOTAL \\
\hline NAME & STAGE & FormHER & LOG \# & UNIT & $2 \%$ & $10 \%$ & $30 \%$ & $50 \%$ & $70 \%$ & $83 \%$ & $90 \%$ & $95 \%$ & $98 \%$ & $100 \%$ \\
\hline LOLPE & 4 & & 1 & $\mathrm{Lb} / \mathrm{A}$ & & & 0.11 & 0.18 & 0.25 & 0.30 & & & & \\
\hline POATR & 4 & & 1 & $\mathrm{Lb} / \mathrm{A}$ & & & & & & & & 0.09 & 0.12 & 0.24 \\
\hline LOLPE & 2 & & 2 & $\mathrm{Lb} / \mathrm{A}$ & & & 0.08 & 0.17 & 0.30 & & & & & \\
\hline POATR & 2 & & 2 & $\mathrm{Lb} / \mathrm{A}$ & & & & & & 0.10 & 0.14 & 0.19 & 0.28 & \\
\hline LOLPE & 27 & & 5 & $\mathrm{Lb} / \mathrm{A}$ & 0.04 & 0.18 & 0.29 & & & & & & & \\
\hline $\begin{array}{l}\text { POATR } \\
\text { fenoxapr }\end{array}$ & rop & & 5 & $\mathrm{Lb} / \mathrm{A}$ & & & & & & & & & 0.11 & 0.24 \\
\hline
\end{tabular}

\section{Possible alternatives}

Additional criteria for searches could be specified outside of the menu-driven shell of the program, by a user familiar with and possessing RBASE for $\operatorname{DOS}^{3}$ command language. Text file output from the database program can be imported into word processors or other programs for further sorting, editing, or viewing. As subsets of an entire weed control database, text file output from this program could provide smaller, more manageable collections of information for use with specific audiences or on computers possessing smaller amounts of available storage space.

\section{System limitations}

This format for a weed control database implements only a limited set of all possible desirable features. Extending the structural framework to include other relevant factors such as soil type, meteorological conditions, and registration status of possible treatments would certainly be a worthwhile endeavor. The primary contribution of this current version is its description of a system to convert raw data into standardized forms that facilitate tabular presentation and comparisons between species and tests. The four equations

\footnotetext{
${ }^{3}$ Microrim Inc., 3925 159th Ave. N.E., P.O. Box 97022, Redmond, WA 99073-9722.
} 
used to describe the dose/response relationship may not provide as good a fit to the data as could be obtained with more complex models available in dedicated data analysis programs. However, the program was not intended to compete with them in analyzing those experiments specifically designed to generate dose/response equations over the full range from no effect up to complete control. Rather, it was designed to work effectively with results from typical field experiments in which herbicides are tested at only a limited number of rates, which may be as few as one and is seldom more than three or four. The issue of the accuracy or validity of the dose/response equation is minimized by limiting its use to estimating rates for specific levels of control only in those categories near the actual data points.

\section{Hardware/software requirements}

The weed control database system was developed for use under $\operatorname{MSDOS}^{4} 3.3$ on an IBM $^{5}$ PC-XT-compatible computer with $640 \mathrm{kB}$ of RAM and hard disk storage using the RBASE for $\mathrm{DOS}^{3}$ programming language. Use of the weed control database system requires either RBASE for DOS or the RBASE RUNTIME ${ }^{3}$ system. It would also be possible for experienced programmers to translate the source code into other database languages. Menu-driven access to the weed control database was developed using the application EXPRESS ${ }^{3}$ program within RBASE for DOS. Separate programs written in the text editor and debugged in RBASE were combined into the general menu using application $\mathrm{CODELOCK}^{3}$. Additional means to search and access the database and format the output could be developed as separate programs and added to the general menu. Database size is limited by hard disk storage capacity. Each single herbicide treatment/plant species entry in the table CNTLRATE occupies a minimum of 164 bytes of disk space, plus some fraction of the 254 byte overhead from the test description entry in the table ENTRYLOG.

To obtain a version of the database program capable of running within RBASE for DOS along with ASCII source code and a sample database, send a blank, formatted, high-density 5.25-inch or 3.5-inch diskette to the author. RBASE for DOS would need to be purchased separately from Microrim Inc. in order to run the database weed control program. A limited number of RUNTIME versions are also available for distribution. ASCII source code is included on the diskette for those who lack RBASE for DOS and cannot use the executing version of the program, but who may wish to translate the code into another database language. TSR programs CAPTURE.COM, SNIPPER.COM, and HELP.COM are included free of charge to enable use and upgrading of the on-line help system.

\footnotetext{
${ }^{4}$ MSDOS is a registered trademark of Microsoft Corp., 16011 NE 36th Way Box 97017, Redmond, WA 98073.

${ }^{5}$ IBM is a registered trademark of International Business Machines Corp., P.O. Box 1328, Boca Raton, FL 33432.
}

Page 11 of 12 


\section{Literature cited}

1. Brain, P. and R. Cousens. 1989. An equation to describe dose responses where there is stimulation of growth at low doses. Weed Pes. 29:93-96.

2. Burrill, L. C., W. S. Braunworth, Jr., R. D. William, R. Parker, D.G. Swan, and D. W. Kidder. 1988. Pacific Northwest Weed Control Handbook. Ext. Serv., Oregon State Univ., Washington State Univ., and Univ. of Idaho. Corvallis, OR and WA. 264 p.

3. Kihlken, T. 1988. Utilities, Capture your own help screens. PC Magazine 7(1):325-337.

4. Linker, H. M, A. C. York, and D. R. Wilhite, Jr. 1990. WEEDS -A system for developing a computerbased herbicide recommendation program. Weed Technol 4:380-385.

5. Little, T. M., and F. J. Hills. 1978. Curvilinear Relationships, Chapter 14 in Agricultural Experimentation. Design and Analysis. John Wiley and Sons, New York.

6. Lutman, P.J.W., and G. G. Tucker. 1987. Standard descriptions of growth stages of annual dicotyledonous weeds. Ann. Appl. Biol 110:683-687.

7. McAllister, R. S. 1990. Electronic Data Exchange Format (EDE 3.0). Nat. Agric. Chem. Assoc., 1155 Fifteenth St., N.W., Suite 900, Washington, DC 20005.

8. Mefford, M. J. 1988. PC Lab Notes, Custom Design Your Own Help Screens. PC Magazine 7(1):311320.

9. Robson, M. J., G.J.A. Ryle, and J. Woledge. 1988. The grass plant - its form and function p. 25-83 in M. B. Jones and A. Lazenby, ed. The Grass Crop -- The Physiological Basis of Production. Chapman and Hall, New York.

10. Zadoks, J. C., T. T. Chang, and C. P. Konzak. 1974. A decimal code for the growth stages of cereals. Weed Res. 14:415-421. 